\title{
Relación entre dos métodos de detección del celo y eficiencia reproductiva en vacas Holstein
}

\author{
Relationship between two methods of heat detection and reproductive \\ efficiency in Holstein cows \\ Yaneline Hidalgo V. ${ }^{1}$, Carlomagno Velásquez V., ${ }^{1}$, Néstor Chagray A. ${ }^{1}$, \\ Nidia Llapapasca G. ${ }^{2}$, Alfredo Delgado C.,3
}

\section{Resumen}

El objetivo del estudio fue evaluar dos métodos de detección del celo en dos épocas del año y su influencia sobre la eficiencia reproductiva de vacas Holstein. Se evaluaron 1445 registros de un establo comercial de explotación intensiva ubicada en el norte de Lima, Perú en 2014. Los métodos de detección de celo fueron un medidor de actividad comercial $\left(\mathrm{T}_{1}\right)$, la observación visual $\left(\mathrm{T}_{2}\right)$ y la combinación de ambos métodos $\left(\mathrm{T}_{3}\right)$. Los parámetros reproductivos evaluados fueron la tasa de detección de celos, la tasa de concepción, el número de servicios por concepción, los días abiertos y el número de parto. El mayor número de celos fue detectado con $\mathrm{T}_{3}(52.2 \%)$ en comparación al $27.5 \%$ de $\mathrm{T}_{1}$ y al 20.2\% de $\mathrm{T}_{2}(\mathrm{p}<0.05)$. $\mathrm{T}_{1}$ fue más eficiente en la detección de celos durante el verano $\left(34.7 \%\right.$ de celos) en comparación con $\mathrm{T}_{2}(13.2 \%)(\mathrm{p}<0.05)$. $\mathrm{T}_{1}$ detectó mayor número de celos en la primera y segunda lactación (30.3 y $26.5 \%$, respectivamente) en comparación con $\mathrm{T}_{2}(20.0$ y $19.7 \%$, respectivamente $)(\mathrm{p}<0.05)$. No hubo diferencia significativa entre tratamientos con relación a la tasa de concepción y número de servicios por concepción; sin embargo, se encontró un menor número de días abiertos en servicios hechos en celos detectados por observación visual (117 días) en comparación con aquellos por el medidor de actividad (144 días). Se concluye que el medidor de actividad es más eficiente que la observación visual en la detección de celos; sin embargo, los mejores resultados se obtienen combinando ambos métodos.

Palabras clave: celo; vacas; detección del celo: medidor de actividad

${ }^{1}$ Facultad Zootecnia, Universidad Nacional Faustino Sánchez Carrión, Huacho, Lima, Perú

${ }^{2}$ Clínica de Animales Mayores, Facultad de Medicina Veterinaria, Universidad Nacional Mayor de San Marcos, Lima, Perú

${ }^{3}$ E-mail: aldelgadoc@gmail.com

Recibido: 7 de junio de 2017

Aceptado para publicación: 17 de octubre de 2017 
The objective of the study was to evaluate two methods of detection of oestrus in two seasons and their influence on reproductive efficiency in Holstein cows. A total of 1445 records was evaluated from an intensive commercial farm located in the north of Lima, Peru in 2014. The methods of detection of oestrus were a commercial activity meter $\left(\mathrm{T}_{1}\right)$, visual observation $\left(\mathrm{T}_{2}\right)$ and the combination of both methods $\left(\mathrm{T}_{3}\right)$. The reproductive parameters evaluated were the rate of heat detection, conception rate, number of services per conception, open days and parity number. The highest number of heats was detected with $\mathrm{T}_{3}(52.2 \%)$ compared to $27.5 \%$ of $\mathrm{T}_{1}$ and $20.2 \%$ of $\mathrm{T}_{2}(\mathrm{p}<0.05)$. $\mathrm{T}_{1}$ was more efficient in detecting heats during the summer $(34.7 \%$ heats $)$ compared to $\mathrm{T}_{2}(13.2 \%)(\mathrm{p}<0.05)$. $\mathrm{T}_{1}$ showed a higher number of heats in the first and second lactations $(30.3$ and $26.5 \%$, respectively) compared to $\mathrm{T}_{2}(20.0$ and $19.7 \%$, respectively) $(\mathrm{p}<0.05)$. There was no significant difference between treatments in relation to conception rate and number of services per conception; however, a lower number of open days in services made in heats detected by visual observation (117 days) were found compared to those by the activity meter (144 days). It is concluded that the activity meter is more efficient than the visual observation in the detection of heats; however, the best results are obtained by combining both methods.

Key words: oestrus; cows; heat detection; activity meter

\section{INTRODUCCIÓN}

La mejora de los niveles de producción de leche en el ganado bovino está derivando en una disminución de las tasas de los parámetros reproductivos (Sepúlveda y Rodero, 2003), debido a causas multifactoriales, siendo entre otras el consumo de alimento, el balance energético negativo (BEN) durante el posparto inmediato y los niveles elevados de proteína en la dieta (Glauber, 2013). Cuando se incrementa la ingestión de materia seca para la mejora de los niveles de producción de leche, la eficiencia reproductiva puede disminuir por causas puramente fisiológicas como consecuencia del metabolismo hepático exacerbado de las hormonas esteroideas (Bach, 2001; Sangsritavong, 2002). Una de las variables reproductivas influenciada por el nivel de producción lechera es la duración e intensidad del celo (López et al., 2004; Glauber, 2013).
La rentabilidad económica de una granja lechera se basa, en parte, en el intervalo entre partos de las vacas. El intervalo óptimo es de 365 días, donde la vaca requiere quedar gestante dentro de los 85 días posparto (Lyimo et al., 2000). Uno de los problemas que se observan en los establos lecheros para poder lograr gestaciones tempranas es la baja tasa de detección de celo, la cual afecta la tasa de servicios, alargando el intervalo parto-concepción y el intervalo entre partos.

El método tradicional de detección del celo es mediante la observación visual. La eficiencia de este método varía entre $45 \%$ (Wainstein et al., 2001) hasta 91\% (Perry, 2005). La eficiencia de este método dependerá de la habilidad del operador de reconocer los signos clínicos del celo. La no observación de actividad de montas está afectada por los periodos cortos de receptividad a la monta, la baja intensidad de actividad, los periodos cortos de observación, y el número de animales en el lote, entre otros (Carmona y Arroyo, 2006). 
Se dispone de una gran variedad de medios auxiliares para la detección de celos. Muchas de ellas son beneficiosas como complemento de un programa de detección visual y no debieran ser considerados como sustitutos de la observación visual (Becaluba F y Becaluba H, 2007). Entre estos se puede mencionar el uso de collares, que registran el nivel de actividad durante el día. La actividad de la vaca en celo aumenta hasta cuatro veces por encima de una línea basal cuando es medida a través de estos monitores. El valor umbral para el incremento de actividad puede ser utilizado para detectar vacas en celo (Matthew, 2009). Este método tiene 70-80\% de eficiencia (Senger, 1994).

Por lo tanto, el objetivo de la presente investigación fue evaluar dos métodos de detección del celo y la combinación de ambos en la eficiencia reproductiva en un establo lechero del valle de Huaura, Perú.

\section{Materiales y Métodos}

El presente trabajo fue de tipo retrospectivo y se realizó con datos del año 2014 proporcionados por el establo lechero «Granados», ubicado en el distrito de Végueta, provincia de Huaura (Lima, Perú). El estudio se realizó durante todo el año, tomando en cuenta como verano el tiempo entre enero y abril (además de diciembre) y como invierno entre mayo y noviembre.

Se evaluaron 568 vacas Holstein de diversa paridad, que tuvieron un registro de 1445 eventos de celo. El sistema de crianza es de tipo intensivo, teniendo una producción promedio de $29.5 \mathrm{~kg}$ leche por vaca/día en tres ordeños por día (08:00, 16:00 y 01:00). El período de espera voluntario fue de 60 días, periodo en que se realiza la revisión de los animales para determinar la completa involución uterina y el reinicio de la ciclicidad ovárica, y se les coloca el collar que monitorea su actividad física.
El método con el que se detecta el celo, así como los servicios efectuados son registrados en el establo. Como métodos de detección del celo fueron la observación visual (OV), el sistema medidor de actividad (MA) y la combinación de ambos métodos (coincide la detección por OV y MA). La observación visual de los celos se realiza cada dos horas, por aproximadamente 40 minutos por lote de vacas, por una persona en el turno de día y por otra en el turno de la noche.

El medidor de actividad es una parte de ALPRO System (DeLaval, Suecia). Este sistema está compuesto por un medidor de actividad que se coloca en un collar alrededor del cuello de la vaca, un receptor con una antena y un procesador que puede conectarse a una computadora para ejecutar el programa ALPRO Windows. El medidor de actividad contiene un sensor que detecta los movimientos, los cuales se registran y se transmiten al receptor cada hora. Cada transmisión contiene datos del último periodo de 24 horas. Así mismo, este medidor tiene una batería (con una vida útil de aproximadamente 10 años) y un sensor electromagnético que detecta los movimientos de la vaca en cualquier dirección. Los collares son colocados en las vacas a los aproximadamente 60 días del parto, teniendo en cuenta el periodo voluntario de espera. Si bien, los collares deberían quedarse colocados en las vacas en forma permanente, el establo solo cuenta con 120 collares, de modo que se les retira cuando se confirma la preñez y se colocan en otras vacas vacías.

Todas las vacas del estudio tenían los collares. En algunos casos el celo fue detectado solo por el collar (T1), en otros solo por la detección visual, pese que las vaca tenían puesto los collares (T2), y en otros casos, el celo fue detectado tanto por el medidor como por la observación visual (T3).

El servicio de todas las vacas se realiza mediante inseminación artificial (IA) con semen importado y con el apoyo de tres 
inseminadores. La IA se realiza 12 horas después de la detección del celo por OV, previa revisión para confirmar el celo. En el caso de celos detectados por el MA, y considerando que el momento óptimo es entre $10 \mathrm{y}$ 28 horas del inicio de la alta actividad, el sistema mostrará la hora de inicio, los reportes son revisados en la mañana y en la tarde, y se realiza la IA según el horario de inicio de alta actividad, previa revisión para confirmar si realmente está en celo.

Para el análisis de los datos se utilizó el programa estadístico MINITAB v. 16. Las comparaciones se llevaron a cabo mediante la prueba de Kruskall-Wallis, ya que los datos no siguen una distribución normal. Se analizaron las variables tasa de concepción, días abiertos, número de servicios por concepción y el porcentaje de celos detectados por cada uno de los métodos, según número de parto y época del año.

\section{Resultados}

Se observó diferencia significativa entre métodos de detección de celo (Cuadro 1; $\mathrm{p}<0.05)$. El medidor de actividad $\left(\mathrm{T}_{1}\right)$ detectó $7.3 \%$ más celos que el método de obser-

Cuadro 1. Eficiencia de dos métodos de detección del celo en vacas lecheras criadas en estabulación en la zona de Huaura, Perú

\begin{tabular}{lcc}
\hline \multirow{2}{*}{ Método } & \multicolumn{2}{c}{$\begin{array}{c}\text { Celos } \\
\text { detectados }\end{array}$} \\
\cline { 2 - 3 } & $\mathrm{n}$ & $\%$ \\
\hline $\begin{array}{l}\left.\text { ( } \mathrm{T}_{1}\right) \text { Medidor de } \\
\text { actividad }\end{array}$ & 398 & $27.5^{\mathrm{a}}$ \\
$\begin{array}{l}\left(\mathrm{T}_{2}\right) \text { Observación } \\
\text { visual }\end{array}$ & 292 & $20.2^{\mathrm{b}}$ \\
$\left(\mathrm{T}_{3}\right) \mathrm{T} 1+\mathrm{T} 2$ & 755 & $52.2^{\mathrm{c}}$ \\
\hline Total & 1445 & 100.0 \\
\hline $\mathrm{a}, \mathrm{b}, \mathrm{c}$ Letras diferentes indican diferencia significa- \\
tiva $(\mathrm{p}<0.05)$
\end{tabular}

vación visual $\left(\mathrm{T}_{2}\right)$, mientras que con la combinación de ambos $\left(\mathrm{T}_{3}\right)$ se llegó a detectar 24.7 y $30 \%$ más celos que con $\mathrm{T}_{1}$ y $\mathrm{T}_{2}$, respectivamente.

La tasa de concepción fue de 26.6, 24.0 y $27.8 \%$ para los servicios detectados en celo para $\mathrm{T}_{1}, \mathrm{~T}_{2} \mathrm{y} \mathrm{T}_{3}$, respectivamente, sin diferencia estadística entre tratamientos, dando una tasa de concepción global de $26.7 \%$. Así mismo, el método de detección del celo no influyó sobre el número de servicios de concepción, donde se obtuvo $2.9,2.2$ y 2.7 servicios por concepción para $\mathrm{T}_{1}, \mathrm{~T}_{2} \mathrm{y} \mathrm{T}_{3}$, respectivamente.

Los métodos de detección del celo influyeron sobre los días abiertos ( $\mathrm{p}<0.05)$, donde el mayor periodo se presentó en $\mathrm{T}_{1}(144$ días), tal como se muestra en el Cuadro 2. La eficiencia de la detección del celo según el método empleado fue dependiente de la estación del año. En el verano, el medidor de actividad fue más eficiente en la detección de celos (34.7\%) en comparación a la observación visual $(13.2 \%)(\mathrm{p}<0.05)$, pero ambos métodos presentaron la misma eficiencia durante el invierno (Cuadro 3). Por otro lado, el método combinado $\left(\mathrm{T}_{3}\right)$ fue el más eficiente durante las dos estaciones del año (Cuadro 3).

La eficiencia de los métodos de detección del celo estuvo influenciada por el número de parto. En las dos primeras lactancias se encontraron diferencias significativas entre tratamientos $(\mathrm{p}<0.05)$, donde $\mathrm{T}_{1}$ mostró una mayor efectividad en comparación a $\mathrm{T}_{2}$. sin embargo, $\mathrm{T}_{3}$ mostró una mayor efectividad en comparación a $\mathrm{T}_{1} \mathrm{y} \mathrm{T}_{2}$ (Cuadro 4). A partir de la tercera lactancia no hubo diferencia significativa entre $\mathrm{T}_{1} \mathrm{y} \mathrm{T}_{2}$.

\section{Discusión}

La proporción de celos detectados con solo el MA fue de $57.7 \%$ y solo con OV fue de $42.3 \%$. La mayor eficiencia en la detección de celo con el método del MA se debe a 
Cuadro 2. Tasa de concepción y número de días abiertos en vacas detectadas en celo por dos métodos de detección del celo en vacas lecheras criadas en estabulación en la zona de Huaura, Perú

\begin{tabular}{lcccc}
\hline Métodos de detección & $\begin{array}{c}\text { Celos } \\
\text { detectados }\end{array}$ & $\begin{array}{c}\text { Tasa de } \\
\text { concepción }\end{array}$ & Días abiertos & $\begin{array}{c}\text { Intervalo de } \\
\text { confianza }\end{array}$ \\
\hline Medidor de actividad & 398 & $26.6^{\mathrm{a}}$ & $144.0^{\mathrm{a}}$ & $129-157$ \\
Observación visual & 292 & $24.0^{\mathrm{a}}$ & $145.3^{\mathrm{a}}$ & $126-164$ \\
Medidor + observación & 755 & $27.8^{\mathrm{a}}$ & $152.9^{\mathrm{a}}$ & $142-164$ \\
\hline Total & 386 & 26.7 & & \\
\hline
\end{tabular}

Superíndices iguales indican que no hubo diferencia significativa entre grupos

Cuadro 3. Eficiencia de dos métodos de detección del celo en vacas lecheras criadas en estabulación en la zona de Huaura, Perú, según la época del año

\begin{tabular}{ccccccccc}
\hline \multirow{2}{*}{ Época } & \multicolumn{2}{c}{$\begin{array}{c}\text { Medidor de } \\
\text { actividad }\end{array}$} & \multicolumn{2}{c}{$\begin{array}{c}\text { Observación } \\
\text { visual }\end{array}$} & \multicolumn{2}{c}{$\begin{array}{c}\text { Medidor }+ \\
\text { observación }\end{array}$} & \multicolumn{2}{c}{ Total } \\
\cline { 2 - 11 } & $\mathrm{n}$ & $\%$ & $\mathrm{n}$ & $\%$ & $\mathrm{n}$ & $\%$ & $\mathrm{n}$ & $\%$ \\
\hline Verano & 186 & $34.7^{\mathrm{a}}$ & 71 & $13.2^{\mathrm{b}}$ & 279 & $52.1^{\mathrm{c}}$ & 536 & 100.0 \\
Invierno & 212 & $23.3^{\mathrm{a}}$ & 221 & $24.3^{\mathrm{a}}$ & 476 & $52.4^{\mathrm{b}}$ & 909 & 100.0 \\
\hline Total & 398 & & 292 & & 755 & & 1,445 & \\
\hline
\end{tabular}

$a, b, c$ Superíndices diferentes entre estaciones indican diferencia estadística $(p<0.05)$

Cuadro 4. Eficiencia de dos métodos de detección del celo en vacas lecheras criadas en estabulación en la zona de Huaura, Perú, según el número de parto

\begin{tabular}{ccccccc}
\hline \multirow{2}{*}{$\begin{array}{c}\text { Numero de } \\
\text { parto }\end{array}$} & \multicolumn{2}{c}{ Medidor de actividad } & \multicolumn{2}{c}{ Observación visual } & \multicolumn{2}{c}{ Medidor + observación } \\
\cline { 2 - 7 } & $\mathrm{n}$ & $\%$ & $\mathrm{n}$ & $\%$ & $\mathrm{n}$ & $\%$ \\
\hline 1 & 161 & $30.3^{\mathrm{a}}$ & 106 & $20.0^{\mathrm{b}}$ & 264 & $49.7^{\mathrm{c}}$ \\
2 & 113 & $26.5^{\mathrm{a}}$ & 84 & $19.7^{\mathrm{b}}$ & 230 & $53.9^{\mathrm{c}}$ \\
3 & 63 & $25.3^{\mathrm{a}}$ & 52 & $20.9^{\mathrm{a}}$ & 134 & $53.8^{\mathrm{b}}$ \\
4 o más & 61 & $25.6^{\mathrm{a}}$ & 50 & $21.0^{\mathrm{a}}$ & 127 & $53.4^{\mathrm{b}}$ \\
\hline Total & 398 & & 292 & & 755 & \\
\hline
\end{tabular}

a,b,c Superíndices diferentes entre número de partos indican diferencia estadística $(p<0.05)$ 
que, bajo condiciones de crianza intensiva, una vaca durante el periodo de celo presenta 2.7 veces más actividad en comparación a una vaca que no está en celo (Senger, 1994); situación que no siempre puede ser detectada a la observación visual.

La tasa de concepción fue similar entre grupos. Según Giudice y Murray (2007), la hora de inicio del celo proporcionado por el sistema MA puede ayudar al ganadero en el momento de decidir la inseminación de la vaca. No obstante, se debe tener en cuenta que los métodos de detección sirven para detectar celos, mientras que la concepción del animal por el servicio efectuado depende de diversos factores; es así, que se puede tener una buena eficiencia en la detección del celo que no vaya acompañada de altas tasas de concepción. La tasa obtenida en el presente estudio (27.6\%) fue baja, probablemente debido al efecto de un verano extremadamente largo para el año en estudio, así como por el efecto de falsos celos que pudieron ser detectados por los métodos empleados.

El número de servicios por concepción y de días abiertos fueron igualmente similares entre tratamientos, lo cual contradice los resultados presentados por Giudice y Murray (2007), quienes encontraron una reducción en los servicios por concepción de 3.7 a 1.9 utilizando el método de medidor de actividad. Por otro lado, Matthew (2009) afirma que los medidores de actividad (tanto podómetros como collares) aumentan los falsos positivos y Cuatrín et al. (2001) señalan que el medidor de actividad detecta muchos falsos celos, por lo que debería complementarse con otros medios (pinturas en la base de la cola, registros, etc.) a fin de mejorar su precisión.

La detección oportuna y eficiente de los celos es crucial en todo sistema intensivo de producción de leche. Sepúlveda y Rodero (2003) sostienen que una deficiente detección de celos disminuye la producción de leche de la vida productiva del animal y el nú- mero de terneros nacidos por vaca, así como un incremento en el número de días abiertos y en la tasa de reposición por problemas reproductivos.

Las diferencias encontradas en la detección de celo por efecto del verano a favor del medidor de actividad discrepan de los resultados hallados por Peralta et al. (2005), quienes obtuvieron una mayor eficiencia en la detección de celo en verano con la observación visual $(49.3 \%)$ en comparación al $37.2 \%$ con el medidor de actividad (ALPRO). No obstante, en ambos casos se confirma la premisa de que la combinación de múltiples sistemas de detección mejora la eficiencia de la detección del celo.

La causa más probable de la disminución en la tasa de detección de celo con el método de observación visual pudo haber sido la reducción de la expresión del comportamiento estral debido al letargo físico causado por el estrés calórico durante el verano, pero que no sería afectado por el medidor de actividad. La reducción de la actividad física es una respuesta adaptiva al medio que ayuda al organismo a limitar la producción de calor. Es así que se observa una disminución de la actividad sexual en el verano frente al invierno (4.5 y 8.6 montas, respectivamente) en vacas Holstein en celo (Góngora y Hernández, 2010). Estos autores indican, asimismo, que la zona termo-neutral del ganado de leche se encuentra en un rango de 5 a $21{ }^{\circ} \mathrm{C}$.

Los resultados del presente estudio indicaron una mayor detección de celo con los tres métodos en vacas de primer y segundo parto en comparación con vacas de más de tres partos. Según Nebel (2004), las vacas Holstein se dejaban montar en promedio siete veces y las vaquillonas 17 veces por día. Así mismo, Nebel (2013) menciona 21\% de reducción en la actividad de caminar durante el celo en cada nueva lactancia, mientras Mathew (2009) menciona una mayor actividad de caminar durante el celo en vaquillas que en vacas primerizas, así como una signi- 
ficativa reducción entre primera y subsecuentes lactancias.

\section{Conclusiones}

- La combinación de los métodos medidor de actividad + observación visual mejoró la detección del celo en el establo en comparación con métodos individuales.

- Los métodos de detección de celo en estudio no influyeron sobre la tasa de concepción, número de servicios por concepción, ni en el número de días abiertos.

- El medidor de actividad debe ser considerado como un método de ayuda y no debe reemplazar completamente a la observación visual.

\section{Literatura Citada}

1. Bach A. 2001. La reproducción del vacuno lechero: nutrición y fisiología. En: XVII Curso de especialización FEDNA. España. [Internet]. Disponible en: http:// www.fundacionfedna.org/publicaciones_2001

2. Becaluba F, Becaluba H. 2007. Nuevas tecnologías para el manejo de la detección del celo. [Internet]. Disponible en: https://www.engormix.com/MAganaderia-carne/genetica/articulos/nuevas-tecnologias-manejo-detecciont1626/p0.htm

3. Carmona G, Arroyo, G 2006. Cómo medir la eficiencia reproductiva de su hato lechero. [Internet]. Disponible en: https://www.engormix.com/ganaderialeche/articulos/como-medir-eficienciareproductiva-t26622.htm

4. Cuatrín A, Maciel M, Taverna M, Mongiardino ME, Dick A, Quaino $O$. 2001. Utilización de podómetros para la detección de celos en vacas lecheras. Argentina: Anuario INTA. [Internet]. Disponible en: http://rafaela.inta.gov.ar/ anuario2001/a2001_95.htm
5. Giudece P, Murray R. 2007. Heat detection analysis using an activity meter in grassland systems. DeLaval. [Internet]. Available in : http:// www.milkproduction.com/Library/ Scientific-articles/Reproduction/Heatdetection-analysis-/

6. Glauber C. 2013. ¿Los altos rendimientos en producción lechera afectan la fertilidad del rodeo? Rev Med Vet (B. Aires) 94: 10-16.

7. Góngora A, Hernández A. 2010. La reproducción de la vaca se ve afectada por las altas temperaturas ambientales. Revista U.D.C.A Actualidad \& Divulgación Científica 13: 141-151.

8. López H, Satter LD, Wiltbank MC. 2004. Relationship between level of milk production and estrous behavior of lactating dairy cows. Anim Reprod Sci 81: 209-223. doi: 10.1016/j.anireprosci.2003.10.009

9. Lyimo ZC, Nielen M, Ouweltjes W, Kruip TA, Eerdenburg FJ. 2000. Relationship between estradiol, cortisol and intensity of estrus behavior in dairy cattle. Theriogenology 53: 1783-1795.

10. Matthew L. 2009. Celo: biología básica y mejoramiento de la detección. Sitio argentino de producción animal. [Internet]. Disponible en: http:// www.produccion-animal.com.ar/informacion_tecnica/inseminacion_artificial/ 161-celo_biologia.pdf

11. Nebel R. 2004. Detección del celo. Debemos cambiar de criterio. [Internet]. Disponible en http://www.produccionanimal.com.ar/informacion_tecnica/ inseminacion_artificial/ 66 deteccion_celo_criterio.pdf

12. Nebel R. 2013. Detección de celos y tiempo de inseminación. [Internet]. Disponible en http://www.engormix.com/ MA-ganaderia-leche/genetica/articulos/ deteccion-celos-tiempo-inseminaciont4613/103-p0.htm

13. Perry G. 2005. Comparison of the efficiency and accuracy of three estrous detection methods to indicate ovulation in beef cattle. South Dakota Beef 
Report. [Internet]. Disponible en: http:// openprairie.sdstate.edu/sd_beefreport_2005/25/

14. Peralta OA, Pearson RE, Nebel RL. 2005. Comparison of three estrus detection systems during summer in a large commercial dairy herd. Anim Reprod Sci 87: 59-72. doi: 10.1016/ j.anireprosci.2004.10.003

15. Sangsritavong S, Peines DK, Sartori R, Armentano LE, Wiltbank MC. 2002. High feed intake increases liver blood flow and metabolism of progesterone and estradiol-17 beta in dairy cattle. J Dairy Sci 85: 2831-2842. doi: 10.3168/jds.S0022-0302(02)74370-1

16. Senger PL. 1994. The estrus detection problem: new concepts, technologies, and possibilities. J Dairy Sci 77: 27452753. doi: 10.3168/jds.S0022-0302(94)77217-9

17. Sepúlveda N, Rodero E. 2003. Comportamiento sexual durante el estro en vacas lecheras. Interciencia 28: 500-503.

18. Wainstein AG, Bernal AS, Iriondo MR, Luco AO. 2001. Heatwatch, sistema electrónico de detección de celo evaluado en hembras Holstein Friesian. Arch Zootec 50: 403-406. 